\title{
Pattern of antibiotic use in the community: Non-adherence and self-prescription rates in an Italian urban population
}

\author{
G. GROSSO, S. MARVENTANO, R. FERRANTI and A. MISTRETTA \\ Department 'G.F. Ingrassia', Hygiene and Public Health, University of Catania, I-95123 Catania, Italy
}

Received December 1, 2011; Accepted February 23, 2012

DOI: $10.3892 / \mathrm{mmr} .2012 .818$

\begin{abstract}
The aim of the present study was to assess the pattern of antibiotic use in a community setting of an urban area of Italy and identify factors that affect adherence to their use. By using a questionnaire-based survey, we collected 1,269 interviews and performed analysis on those patients who had their last course of antibiotic within the past 12 months (956 subjects). Among the subjects reporting that they had not followed their last antibiotic course as prescribed, $14.7 \%$ stopped therapy early, $5.4 \%$ modified the dosage, and $5 \%$ changed the prescribed antibiotic. Approximately $23 \%$ of the subjects declared that they self-prescribed antibiotics. After adjusting for all covariates, major predictors for the selfprescription of antibiotics were younger age, female gender and higher socioeconomic and educational status. Conversely, both low educational and socioeconomic status were associated with a higher risk of non-adherence to physician indications. The findings of this study assessed the widespread pattern of poor antibiotic-taking behavior and provides important implications for understanding the targets of future educational campaigns to control the use and misuse of antibiotics.
\end{abstract}

\section{Introduction}

Non-adherence is defined as the extent to which an individual's behavior (taking medication, following a diet and/or executing lifestyle changes) corresponds with the agreed recommendations of a healthcare provider (1). Specifically, non-adherence to medication is defined as the attitude of a patient for not taking a prescribed medication or following a prescribed course of therapy. Although non-adherence to medication is often related to chronic conditions, a significant percentage of patients are not fully compliant with basic medication for acute diseases, including antibiotics. Antibiotics are among the most commonly used medicines worldwide. Italy has the third highest number

Correspondence to: Dr Stefano Marventano, Department 'G.F. Ingrassia', Hygiene and Public Health, University of Catania, Via S. Sofia 85, I-95123 Catania, Italy

E-mail: stefanomarv@gmail.com

Key words: antibiotic, adherence, self-prescribe of antibiotic prescriptions in Europe (2). Data collected by the National Observatory on the Use of Medicines (Osservatorio Nazionale Sull'impiego dei Medicinali, OsMED) reported that in Italy the cost of antibiotics amounts to 1,204 million euros in the community setting and 1,265 million euros in public healthcare structures. Antibiotics account for the fourth leading medicine in terms of expenditures and ninth for prescribed quantities. They are mostly prescribed in the marginal range of age - less than 4 years and more than 65 years - but in recent years there has been a progressive increasing usage in subjects between 15 and 64 years of age. Finally, throughout the country, there is a high regional variability in the number of antibiotic prescriptions and a consistent geographic trend with a deviation from the national average by more than $20 \%$ in the southern regions and islands (3). Despite their importance, improper use of antibiotics contributes to the emergence of antimicrobial resistance. In fact, the phenomenon of resistance has been attributed both to indiscriminate overuse and misuse of antibiotics $(4,5)$. At present, antibiotic resistance is one of the major public health threats worldwide leading to antimicrobial drug-resistant strains and making bacterial infections more difficult to treat (6-10).

Poor adherence to antibiotic regimens has resulted in one of the major causes of treatment failure. Non-adherence to antibiotic treatment has been widely demonstrated to have a profound negative impact on patient health resulting in the reduction of clinical success (11), additional physician visits, extra drug prescriptions and hospital admissions with an increase in additional costs to manage non-adherent patients (12). Intervention to reduce antibiotic misuse must include both patient and physician education concerning the appropriate use of antibiotics. Nevertheless, before starting an intervention program, public health workers need to know the impact of the phenomenon in the community setting in which the intervention should be addressed, and to identify the characteristics of the population related to the attitude of non-adherence.

The present study was aimed at deepening our knowledge of patient non-adherence with antibiotic treatment in an Italian urban setting and identifying patient characteristics that predict a worse compliance. Specifically, the study focused on self-prescription and the use of recycled antibiotics as well as determining which subjects in our community do not follow antibiotic regimens as prescribed. We also evaluated factors associated with such antibiotic abuse and misuse. By iden- 
tifying the target population, more appropriate educational support can be implemented and more effective interventions aimed at improving patient compliance may be designed.

\section{Subjects and methods}

Study population. The study survey was carried out in Catania (approximately 300,000 inhabitants), a city on the east side of Sicily, Italy, from October 2009 through October 2010. Information was recorded from structured, closed-question data forms. The population included in the study was selected by a randomized sampling of 13 outpatient clinics with general practitioners. All clinics were located in urban areas, serving a total population of approximately 19,000 patients. Each district of the city was involved in distributing the questionnaires to at least one outpatient clinic depending on the number of inhabitants of the served zone.

Data collection. Questionnaires were developed to collect information related to demographics and the most recent use of antibiotics within the last year. Demographic data in the first part of the questionnaire included age, gender, level of education and occupation. With regard to employment status, we used codes supplied by the Office for National Statistics (ONS) to characterize the professional status of the subjects in 4 groups as shown below: i) unemployed and housewives, ii) unskilled occupations, iii) partly skilled and skilled occupations, and iv) professional and managerial occupations. Educational level included 3 groups according to the highest level of education attained by the subject: i) none, elementary or secondary school, ii) high or technical school, and iii) university.

The second part of the questionnaire posed questions concerning the cause leading to the most recent antibiotic intake within the past 12 months. Only the data of respondents that used antibiotics within the last year were considered eligible for analysis. Participants were asked questions concerning the relative adherence with their last treatment course (i.e. Did you take the required number of daily doses over the full duration of the course? Did you modified the dosage? Did you change the antibiotic?) as well as questions aimed to assess the use of self-prescribed antibiotics without the advice of a physician (i.e. Did you take antibiotics without a prescription? Did you follow the advice of your doctor?). The questionnaires were anonymous and verbal consent was obtained from all the subjects before compiling the data.

Outcome measures. We used the term 'adherence' to refer to the degree of conformity with the indications of the physician (length of the treatment course, number of doses, and type of antibiotic) and the term 'self-prescribe' to refer to the behavior of a patient to use antibiotics without advice from the doctor. We analyzed the demographic characteristics affecting nonadherence with prescribed antibiotics or self-prescription to identify potential predictors of poor compliance. We defined 'non-compliant' as a subject who self-prescribed antibiotics and/or who did not adhere to the physician's prescription.

Statistical analysis. Categorical data were presented as frequency of occurrence. The Chi-square test for categorical data was used to examine bivariate relationships between
Table I. Demographics and pattern of antibiotic use of the study population $(\mathrm{n}=956)$.

Respondents

$\% \quad \mathrm{n}$

Antibiotic use within the last year

Respiratory tract infections

Gastrointestinal tract infections

59.7

571

Infections at other sites

16.2

155

Age

$<25$ years

$24.1 \quad 230$

25-44 years

10.1

45-65 years

24.0

$>65$ years

36.3

Gender

Male

29.6

Female

43.1

412

Level of education

Elementary or secondary

52.5

502

High school

28.9

276

University

18.6

178

Occupation

Unemployed

20.2

193

Unskilled occupations

Partly skilled occupations

Skilled occupations

Non-compliant patients

Self-prescribed antibiotics

Did not consult the physician

Non-adherence to physician indications

Did not complete the course

Modified the drug dosage

Changed the drug

5.4

self-prescribing and non-adherence according to demographic characteristics. A stepwise logistic regression model was computed for the dependent variables of self-prescription and non-adherence to the antibiotic course with the same set of variables to determine the best predictors of these behaviors. Multivariate regression was used to calculate the odds ratios (ORs) for the trend to self-prescribe antibiotics and not adhere to physician indications where 95\% confidence intervals (CIs) were based on the estimated standard errors. All tests were two-tailed with $\alpha$ levels of 0.05 considered significant.

Data were entered into Microsoft Excel for Windows (Microsoft Corporation, Redmond, WA, USA). Statistical analysis was performed using SPSS for Windows release 17.0 (SPSS Inc., Chicago, IL, USA).

\section{Results}

Eleven of the 13 general practitioners agreed to participate in the study resulting in a total of 1,269 subjects completing the 
Table II. Self-prescription and non-adherence to antibiotic use with respect to the study variables.

\begin{tabular}{|c|c|c|c|c|c|c|}
\hline & \multicolumn{3}{|c|}{ Self-prescription } & \multicolumn{3}{|c|}{ Non-adherence to prescription } \\
\hline & $\begin{array}{c}\text { No }(\mathrm{n}=716) \\
\%(\mathrm{n})\end{array}$ & $\begin{array}{c}\text { Yes }(\mathrm{n}=217) \\
\%(\mathrm{n})\end{array}$ & P-value & $\begin{array}{c}\text { No }(\mathrm{n}=709) \\
\%(\mathrm{n})\end{array}$ & $\begin{array}{c}\text { Yes }(\mathrm{n}=247) \\
\%(\mathrm{n})\end{array}$ & P-value \\
\hline Age, $\%(\mathrm{n})$ & & & $<0.001$ & & & 0.226 \\
\hline$<25$ years & $10.9(78)$ & $8.3(18)$ & & $9.2 \quad(65)$ & $13.0(32)$ & \\
\hline $25-44$ years & $19.7(141)$ & $39.6(86)$ & & $23.4(166)$ & 25.5 & \\
\hline $45-65$ years & $35.1(251)$ & $38.7(84)$ & & $37.7(267)$ & $32.4(80)$ & \\
\hline$>65$ years & $34.3(246)$ & $13.4(29)$ & & $29.8(211)$ & $29.1(72)$ & \\
\hline Gender, \% (n) & & & $<0.001$ & & & 0.947 \\
\hline Male & $46.6(334)$ & $31.8 \quad(69)$ & & $43.2(306)$ & $42.9(106)$ & \\
\hline Female & $53.4(382)$ & $68.2(148)$ & & $56.8(403)$ & $57.1(141)$ & \\
\hline Level of education, $\%$ (n) & & & $<0.001$ & & & $<0.001$ \\
\hline Elementary or secondary & $56.8(407)$ & $37.8(82)$ & & $47.0(333)$ & $68.4(169)$ & \\
\hline High school & $28.9(207)$ & $28.1(61)$ & & $32.6(231)$ & 18.2 & \\
\hline University & $14.3(102)$ & $34.1(74)$ & & $20.5(145)$ & 13.4 & \\
\hline Occupation, \% (n) & & & $<0.001$ & & & $<0.001$ \\
\hline Unemployed & $22.1(158)$ & $12.9(28)$ & & $18.3(130)$ & $25.5(63)$ & \\
\hline Unskilled occupations & $17.6(126)$ & $9.7 \quad(21)$ & & $11.8 \quad(84)$ & $25.5(63)$ & \\
\hline Partly skilled occupations & $47.1(337)$ & $51.6(112)$ & & $51.8(367)$ & $38.9(96)$ & \\
\hline Skilled occupations & 13.3 & $25.8 \quad(56)$ & & $18.1(128)$ & $10.1(25)$ & \\
\hline
\end{tabular}

questionnaire. Nine hundred and fifty-six respondents $(\sim 75 \%)$ reported that they had been prescribed an antibiotic within the last year. The demographic characteristics of the subjects that reported antibiotic use during the last year and their patterns of use are shown in Table I. Those respondents which were prescribed an antibiotic in the last year were women, older than 45 years and with a lower level of education. The majority of the respondents completed the course $(\sim 85 \%)$. Among those reporting that they did not follow their last antibiotic course as prescribed, $14.7 \%$ stopped therapy early, $5.4 \%$ modified the dosage, and 5\% changed the prescribed antibiotic. Moreover, approximately $23 \%$ of the subjects declared self-prescription of antibiotics and $37 \%$ of them did not consult a physician after using them. Thus, a total of $45 \%$ of patients were noncompliant with antibiotic therapy.

Characteristics of the study population in respect to their non-compliant attitude (namely self-prescription and non-adherence with physician indications) are presented in Table II. Respondents more likely to self-prescribe antibiotics were middle class female with a higher educational level and occupational status $(\mathrm{P}<0.001)$. On the other hand, subjects who did not adhere to physician indications were found to have a lower level of education and occupational status $(\mathrm{P}<0.001)$. No statistically significant relation was found for gender and age.

The multivariate logistic regression model is shown in Table III. After adjusting for all covariates, the major predictors for self-prescription of antibiotics were younger age, female gender and higher educational level and occupational status. Specifically, a higher risk for the self-prescription of antibiotics was found for patients 25-44 years of age (HR, 5.01; 95\% CI, 3.06-8.19; $\mathrm{P}<0.001)$, while subjects with an elementary school education and those with unskilled occupations had a lower risk (HR, 0.39; 95\% CI, 0.26-0.61; $\mathrm{P}<0.001$ and HR, 0.35; 95\% CI, 0.19-0.65; $\mathrm{P}=0.001$, respectively).

With regards to adherence to physician indications, the multivariate logistic regression model revealed that both low educational level and occupational status were associated with a higher risk of non-adherence. In detail, patients who completed elementary school had a higher risk than those who achieved a university degree (HR, 1.77; 95\% CI, 1.13-2.75; $\mathrm{P}=0.012)$ as well as unemployed and subjects with unskilled occupations (HR, 1.82; 95\% CI, 1.04-3.13; $\mathrm{P}=0.035$ and $\mathrm{HR}$, 2.82; 95\% CI, 1.61-4.94; $\mathrm{P}<0.001$, respectively).

\section{Discussion}

This study evaluated variables associated with poor compliance with antibiotics therapy. Results indicated that multiple factors are related to the non-compliance of individuals. Specifically, differences in patient characteristics were found to distinguish between those who did not follow the therapy as prescribed (stopping early, modifying the dosage, or changing the drug) and those who self-prescribed antibiotics.

The study revealed that nearly a quarter of the subjects use non-prescribed antibiotics in the Sicilian urban population. Moreover, a relatively high percentage of respondents discontinued therapy early and admitted to not following the recommended dosage or even to substituting the antibiotic. Several of the most recent reviews reported similar rates compared to our results, revealing that compliance was generally unsatisfactory (13-15). Although there is a substantial heterogeneity in the definition of 'non-compliance', the 
Table III. Significant predictors from stepwise logistic regression for self-prescription and non-adherence to antibiotics.

\begin{tabular}{|c|c|c|c|c|}
\hline \multirow[b]{2}{*}{ Variables } & \multicolumn{2}{|c|}{ Self-prescription } & \multicolumn{2}{|c|}{ Non-adherence with prescription } \\
\hline & OR $(95 \% \mathrm{CI})$ & P-value & OR $(95 \% \mathrm{CI})$ & P-value \\
\hline Age & & & NS & - \\
\hline$<25$ years & $2.25(1.15-4.42)$ & 0.019 & & \\
\hline $25-44$ years & $5.01(3.06-8.19)$ & $<0.001$ & & \\
\hline $45-65$ years & $2.72(1.69-4.39)$ & $<0.001$ & & \\
\hline$>65$ years & 1 & - & & \\
\hline Gender & & & NS & - \\
\hline Male & $0.54(0.38-0.76)$ & $<0.001$ & & \\
\hline Female & 1 & - & & \\
\hline \multicolumn{5}{|l|}{ Level of education } \\
\hline Elementary or secondary & $0.39(0.26-0.61)$ & $<0.001$ & $1.77(1.13-2.75)$ & 0.012 \\
\hline High school & $0.41(0.26-0.64)$ & $<0.001$ & $0.79(0.48-1.31)$ & 0.359 \\
\hline University & 1 & - & 1 & - \\
\hline \multicolumn{5}{|l|}{ Occupation } \\
\hline Unemployed & $0.37(0.21-0.65)$ & 0.001 & $1.81(1.04-3.13)$ & 0.035 \\
\hline Unskilled occupations & $0.35(0.19-0.65)$ & 0.001 & $2.82(1.61-4.94)$ & $<0.001$ \\
\hline Partly skilled occupations & $0.66(0.43-1.02)$ & 0.062 & $1.11(0.67-1.81)$ & 0.693 \\
\hline Skilled occupations & 1 & - & 1 & - \\
\hline
\end{tabular}

results from the large number of studies appear to agree on these rates. However, one of the major challenges regarding this issue remains differences in the measurement methods of non-compliance. In fact, the distinction between predictors of misuse and those of abuse was poorly differentiated, identifying such unhealthy behaviors under the general terms of non-compliance or non-adherence. Yet, collectively, these estimates of compliance showed a widespread pattern of poor antibiotic-taking behavior.

Notably, we found in our population that the subjects who were more likely to abuse antibiotics without consulting a physician and, in contrast, those who were more prone not to follow the drug protocol as prescribed, had opposing characteristics. In fact, individuals with a lower educational level and occupational status tended to misuse antibiotics while those with a higher level tended to abuse drugs. Other studies revealed similar (16) or lower rates (17), but equal predictive factors of self-medicated drug use. One could speculate that the attitude of the patient may reflect the level of knowledge concerning antibiotics. In fact, greater knowledge concerning antibiotics was associated with a greater tendency to keep leftover antibiotics and use them without advice from a clinician. Medical awareness of patients with a high socioeconomic and educational status may play a role in the increased use of non-prescribed antibiotics. Thus, subjects with a higher level of education and a higher socioeconomic status may be more likely to feel confident in diagnosing an illness and, consequently, in self-prescribing an antibiotic therapy. However, the fact that part of such a population consists of doctors should be considered as a possible source of bias since selfprescribing behavior rates may be strongly influenced by their presence.
Conflicting observations have been shown regarding the role of age in non-adherence to antibiotic therapy. Several studies reported that patient age has no impact on adherence (18), while other studies found higher rates of non-adherence of antibiotics in younger (15) and in older patients (19). In the present study, younger subjects were more prone to be less compliant. In fact, in accordance with a global survey, there was a negative correlation between patient age and both selfprescription and non-adherence to the prescription of their physician (15). This may depend on the relationship between the patient and the general practitioner. In fact, younger patients are more likely to be non-compliant as they perceive greater freedom than older patients with regard to the use of drugs and the advice of physicians.

With regard to gender, experts do not agree on the true role of gender in determining attitudes of non-compliance (13). However, our study showed that female gender was a predictive factor of a non-compliant attitude even if this result was due to a greater number of women taking antibiotics in our study population.

Antibiotics are widely used both in the community and in the hospital setting. Nevertheless, their effectiveness is strongly related to how such medications are used by the patients, particularly in the community setting. Previous studies reported that patients admit to discontinuing antibiotic therapy when they begin to feel better or due to the onset of adverse events $(20,21)$. The use of recycled medications and the phenomenon of self-prescribing antibiotics are the severe consequences of poor compliance with antibiotic therapy. In our study, we found higher rates for self-prescription than in poor adherence with prescribed antibiotics. Although leftover antibiotics result from a discontinuation of prior treatment, 
it may also be influenced by the packaging and medication dispensing of the drug. In fact, in countries such as Italy, where drugs require pre-packaged doses of medication, a greater quantity of medication is dispensed than is required, resulting in the availability of leftover doses even after completion of the prescribed regimen (22). Antibiotic resistance is mainly attributed to the indiscriminate overuse of antibiotics yet doctors may also play a role in this issue. Several investigators have reported inappropriate prescribing practices by physicians in the outpatient setting. Indeed, most of the studies revealed that about half of patients with a common cold are often treated with antibiotics $(23,24)$, instead of a more discriminate use (25). The reasons why physicians inappropriately prescribe antibiotics for conditions that can be cured without using such a therapy can be explained by patient expectations (26). In fact, clinicians report that they often prescribe antibiotics since they perceive that patients want them (27). Patient beliefs concerning the expectations for and effectiveness of antibiotics for minor illnesses play a key role in the unnecessary use of antibiotics (28). Although patient expectation and perceived antibiotic efficacy depend on several factors such as the length of treatment course, time to the onset of symptom relief and time to complete resolution of symptoms (29), their attitude about the need for antibiotics often depends on the behavior of their physician. It is not surprising that in a study conducted in the outpatient setting, patients whose doctors routinely prescribed antibiotics for cold symptoms were more prone to use antibiotics without the advice of a physician (30). Finally, diagnostic uncertainty may also be addressed as a cause of inappropriate prescription (31).

The findings in this study have important implications for understanding the targets of future educational campaigns to control the use and the misuse of antibiotics. Results addressed a need for culturally tailored interventions to more effectively educate the population. Interventions to improve adherence designed by public health workers should include counselling on the importance of adherence as well as educational campaigns appropriately tailored to the target population according to the findings assessed in this study. Furthermore, some campaign strategies may include the distribution of educational materials (posters, brochures) educating the population to the correct use of antibiotics, (32) suggesting over-the-counter remedies (33) and relief strategies for viral infections as better therapies (34). In combination with these public health interventions, general practitioners should minimize antibiotic misuse by reducing regimen complexity, including lower dosage frequency and shorter therapy durations (35). Moreover, the use of written instructions and reminder devices to improve adherence and compliance with antibiotic therapy should be implemented and clearly stated. Finally, physician education should be aimed at providing more extensive knowledge to improve the quality of antibiotic selection (36).

This study has some limitations that warrant discussion. First, one limitation may depend on the methodology used to assess non-compliance. In fact, data concerning antibiotic misuse was reliant on patient self-reporting and so the level of non-compliance is likely to be an underestimate of the true phenomenon. However, the objective of the study was not only to estimate the absolute figures of the community, but rather to examine factors that may be associated with patient non-adherence to antibiotic regimens and suggest ways in which these issues can be addressed. Moreover, there are currently no standardized, validated instruments with which to measure adherence to antibiotic therapy and patient-reported adherence is one of the most frequently used methods to obtain subjective estimates.

Another limitation is that patients were asked several questions about their past use of antibiotics which may have led to recall bias. Moreover, given the cross-sectional nature of the study and the use of a questionnaire to further define the scope of patient antibiotic misuse, the possibility that respondents may have misunderstood or misinterpreted questions should be taken in account. In fact patient may not be able to identify whether a medication is an antibiotic or not, contributing to the creation of biases. However, this study had various strengths. The population size was sufficiently representative of the urban setting of the city. Moreover, this study is the only study conducted in Sicily and, also, one of the few in the whole Italian setting. Thus, we consider the recognition of such issues in our country crucial due to the current policy of medication packing that inevitably contributes to the selfprescription of drugs.

Future studies designed on a large-scale and aimed at including a more heterogeneous population mix to be more representative of the whole Italian population are needed. More studies measuring the knowledge and attitudes toward self-prescription practices would also be helpful to design appropriate interventions. Antibiotic use in the community must be improved through targeted intervention aimed at increasing the education of patients and physicians on the appropriate use of antibiotics. Topics dealing with the dangers of using recycled antibiotics and, on the other hand, adherence to physician prescriptions should be addressed to individuals of a higher and lower education level, respectively. However, a general message towards the community aimed at improve compliance with antibiotics is needed. Moreover, the high cost of non-compliance on healthcare expenditure as well as the benefits on patient health, warrant using such interventions.

\section{Acknowledgements}

The authors thank Professor P.M. Furneri (University of Catania) for the important suggestions for the manuscript. The authors declare no sources of support in the form of grants, equipment, drugs, or all of the above. Any commercial relationships that may pose a conflict of interest in connection with the submitted manuscript must be disclosed.

\section{References}

1. Adherence to long-term therapies: evidence for action. World Health Organization [WHO]. http://www.who.int/chronic_conditions/en/adherence_report.pdf. Accessed Dec, 2010.

2. European Surveillance of Antimicrobial Consumption. http:// www.esac.ua.ac.be. Accessed Dec, 2010.

3. Gruppo di lavoro OsMED: L'uso dei Farmaci in Italia. Rapporto nazionale anno 2009. Il Pensiero Scientifico Editore (ed). Roma, pp54-55, 2010.

4. Branthwaite A and Pechere JC: Pan-European survey of patients attitudes to antibiotics and antibiotic use. J Int Med Res 24: 229-238, 1996 
5. Albrich WC, Monnet DL and Harbarth S: Antibiotic selection pressure and resistance in Streptococcus pneumoniae and Streptococcus pyogenes. Emerg Infect Dis 10: 514-517, 2004.

6. Dancer SJ: How antibiotics can make us sick: the less obvious adverse effects of antimicrobial chemotherapy. Lancet Infect Dis 4: 611-619, 2004.

7. Hooton TM and Levy SB: Antimicrobial resistance: a plan of action for community practice. Am Fam Physician 63: 1087-1098, 2001.

8. Levy SB and Marshall B: Antibacterial resistance worldwide: causes, challenges and responses. Nat Med 10: S122-S129, 2004

9. Maskalyk J: Antimicrobial resistance takes another step forward CMAJ 167: 375, 2002.

10. Patient, provider education targeted in efforts to curb antibiotic resistance. Dis Manag Advis 7: 13-16, 2001.

11. Nicolau D: Clinical and economic implications of antimicrobial resistance for the management of community-acquired respiratory tract infections. J Antimicrob Chemother 50 (Suppl 1): S61-S70, 2002.

12. Sorensen SV, Baker T, Fleurence R, Dixon J, Roberts C, Haider S and Hughes D: Cost and clinical consequence of antibiotic nonadherence in acute exacerbations of chronic bronchitis. Int $\mathrm{J}$ Tuberc Lung Dis 13: 945-954, 2009.

13. Kardas P, Devine S, Golembesky A and Roberts C: A systematic review and meta-analysis of misuse of antibiotic therapies in the community. Int J Antimicrob Agents 26: 106-113, 2005.

14. Cespedes A and Larson E: Knowledge, attitudes, and practices regarding antibiotic use among Latinos in the United States: review and recommendations. Am J Infect Control 34: 495-502, 2006.

15. Pechere JC,Hughes D, Kardas P and Cornaglia G: Non-compliance with antibiotic therapy for acute community infections: a global survey. Int J Antimicrob Agents 29: 245-253, 2007.

16. Skliros E, Merkouris P, Papazafiropoulou A, et al: Self-medication with antibiotics in rural population in Greece: a cross-sectional multicenter study. BMC Fam Pract 11: 58, 2010.

17. Carrasco-Garrido P, Jimenez-Garcia R, Barrera VH and Gil de MA Predictive factors of self-medicated drug use among the Spanish adult population. Pharmacoepidemiol Drug Saf 17: 193-199, 2008

18. Imai-Kamata $\mathrm{S}$ and Fushimi K: Factors associated with adherence to prophylactic antibiotic therapy for elective general surgeries in Japan. Int J Qual Health Care 23: 167-172, 2011.

19. Kennedy J, Tuleu I and Mackay K: Unfilled prescriptions of medicare beneficiaries: prevalence, reasons, and types of medicines prescribed. J Manag Care Pharm 14: 553-560, 2008.

20. Pechere JC: Parameters important in short antibiotic courses. J Int Med Res 28 (Suppl 1): S3A-S12A, 2000.

21. Pechere JC: Patients' interviews and misuse of antibiotics. Clin Infect Dis 33 (Suppl 3): S170-S173, 2001.

22. Wright JM, Htun Y, Leong MG, Forman P and Ballard RC: Evaluation of the use of calendar blister packaging on patient compliance with STD syndromic treatment regimens. Sex Transm Dis 26: 556-563, 1999.
23. Gonzales R, Steiner JF and Sande MA: Antibiotic prescribing for adults with colds, upper respiratory tract infections, and bronchitis by ambulatory care physicians. JAMA 278: 901-904, 1997.

24. Nyquist AC, Gonzales R, Steiner JF and Sande MA: Antibiotic prescribing for children with colds, upper respiratory tract infections, and bronchitis. JAMA 279: 875-877, 1998.

25. Stolz D and Tamm M: Discriminate use of antibiotics for exacerbation of COPD. Curr Opin Pulm Med 15: 126-132, 2009.

26. Macfarlane J, Holmes W, Macfarlane R and Britten N: Influence of patients' expectations on antibiotic management of acute lower respiratory tract illness in general practice: questionnaire study. BMJ 315: 1211-1214, 1997.

27. Coenen S, Michiels B, Renard D, Denekens J and Van RP. Antibiotic prescribing for acute cough: the effect of perceived patient demand. Br J Gen Pract 56: 183-190, 2006

28. Butler CC, Rollnick S, Pill R, Maggs-Rapport F and Stott N: Understanding the culture of prescribing: qualitative study of general practitioners' and patients' perceptions of antibiotics for sore throats. BMJ 317: 637-642, 1998.

29. Perez-Gorricho B and Ripoll M: Does short-course antibiotic therapy better meet patient expectations? Int J Antimicrob Agents 21: 222-228, 2003.

30. Richman PB, Garra G, Eskin B, Nashed AH and Cody R: Oral antibiotic use without consulting a physician: a survey of ED patients. Am J Emerg Med 19: 57-60, 2001

31. Moro ML, Marchi M, Gagliotti C, Di MS and Resi D: Why do paediatricians prescribe antibiotics? Results of an Italian regional project. BMC Pediatr 9: 69, 2009.

32. Wutzke SE, Artist MA, Kehoe LA, Fletcher M, Mackson JM and Weekes LM: Evaluation of a national programme to reduce inappropriate use of antibiotics for upper respiratory tract infections: effects on consumer awareness, beliefs, attitudes and behaviour in Australia. Health Promot Int 22: 53-64, 2007.

33. Garbutt JM, Sterkel R, Banister C, Walbert C and Strunk RC: Physician and parent response to the FDA advisory about use of over-the-counter cough and cold medications. Acad Pediatr 10: 64-69, 2010.

34. Cebotarenco N and Bush PJ: Reducing antibiotics for colds and flu: a student-taught program. Health Educ Res 23: 146-157, 2008.

35. Leclercq R: Safeguarding future antimicrobial options: strategies to minimize resistance. Clin Microbiol Infect 7 (Suppl 3): S18-S23, 2001.

36. Steinman MA, Ranji SR, Shojania KG and Gonzales R: Improving antibiotic selection: a systematic review and quantitative analysis of quality improvement strategies. Med Care 44: 617-628, 2006. 